\title{
AERODYNAMIC ROUGHNESS OF A PLANT CANOPY: A NUMERICAL EXPERIMENT
}

\author{
ROGER H. SHAW \\ Department of Land, Air and Water Resources, University of California, Davis, \\ CA (U.S.A.)
}

A.R. PEREIRA

Instituto Agronomico, Campinas, SP 13100 (Brazil)

(Received October 16, 1981; accepted November 3, 1981)

\section{ABSTRACT}

Shaw, R.H. and Pereira, A.R., 1982. Aerodynamic roughness of a plant canopy: a numerical experiment. Agric. Meteorol., 26: 51-65.

A numerical model based on second-order closure principles was used to evaluate the response of the logarithmic wind profile parameters, the roughness length, $z_{0}$ and the displacement height, $d$, to changes in the density and vertical structure of an underlying canopy of vegetation. The profile parameters were calculated by forcing the logarithmic wind equation to match the computed wind profile over three successive grid points used in the numerical model. Both $\mathrm{z}_{0}$ and $d$ calculated in this manner were functions of height but the displacement height calculated from the wind profile at twice the canopy height was a good approximation to the center-of-pressure within the canopy over a wide range of densities. The displacement height increased monotonically with plant density and with the height of the center of gravity of the vegetation. The roughness length was a unimodal function of density, increasing with density in sparse canopies but decreasing with density in dense canopies. Vertical structure was important. The roughest canopies were those with a high center of gravity at low plant densities but those with a low center of gravity at high densities. At high densities, a single linear relationship between $\mathrm{z}_{0}$ and $d$ was evident, irrespective of density or structure.

Evidence based on the sensitivity of the profile parameters to an arbitrarily set length scale suggests that a second-order closure model is superior to the traditional gradientdiffusion model in the proximity of a plant canopy.

\section{INTRODUCTION}

The aerodynamic drag on the lowest layers of the atmosphere depends on the structure and density of the elements of which the underlying surface is composed. For vegetated surfaces, the roughness will be determined by the height of the canopy, the structure and flexibility of individual plants, the size and arrangement of plant parts, and the planting density.

Relationships between the drag force and the structure of the surface may be sought by direct field observation, by wind tunnel modeling in which attempts are made to achieve some degree of geometric and dynamic similarity, and by computational modeling. This report contains results of a numerical model employing second-order closure principles, and in which roughness 
was evaluated in terms of canopy structure and density. A numerical model has a clear economic gain over field or wind tunnel experiments, while second-order turbulence closure holds definite advantages over the traditional gradient-diffusion or mixing length theories in relatively complex flow configurations such as exist in the proximity of a plant canopy. A numerical model has value in the performance of sensitivity analyses and in the interpretation of actual observations.

\section{WIND PROFILE PARAMETERS AND SURFACE PROPERTIES}

The surface shear stress, $\tau_{0}$, is commonly related to surface properties by a drag coefficient $C_{\mathrm{D}}$, which is usually defined in meteorological literature by

$\tau_{0}=\rho C_{\mathbf{D}} U^{2}$

where $\rho$ is the density of air, and $U=U(z)$ is the mean wind speed at some specified height $z . \tau_{0}$ may be written as $\rho \mathbf{u}_{*}^{2}$, defining a friction velocity $\mathbf{u}_{*}$, in which case

$C_{\mathrm{D}}=\mathbf{u}_{*}^{2} / U^{2}$

The drag coefficient is, in general, only weakly dependent on wind speed and is not greatly influenced by thermal stratification if $U$ is measured at a relatively small height. On the other hand, $C_{\mathrm{D}}$ is a function of height above the surface because the reference height at which the wind speed is measured resides within the boundary layer of surface influence, and therefore $C_{\mathrm{D}}$ loses much of its appeal. This height dependence can be eliminated if the form of the wind profile is known, although the resulting drag parameter will no longer be dimensionless.

When the only length scale to enter the problem is the distance from the solid boundary, simple dimensional reasoning leads to the familiar logarithmic profile. In integrated form, this is commonly expressed as

$U=\left(\mathbf{u}_{*} / \mathrm{k}\right) \ln \left(z / \mathbf{z}_{0}\right)$

where $\mathrm{k}$ is von Karman's constant and $z_{0}$ is an integration constant with the dimension of length. $z_{0}$ relates the friction velocity $\mathbf{u}_{*}$ to the mean wind speed $U$ measured at height $z$. Combining eqs. 2 and 3 yields

$C_{\mathrm{D}}=\left\{\mathrm{k} /\left[\ln \left(z / \mathrm{z}_{0}\right)\right]\right\}^{2}$

Thus, $z_{0}$ increases monotonically with $C_{\mathrm{D}}$. (and vice versa) and, when the mean wind profile matches eq. $3, z_{0}$ is independent of height and becomes a useful parameter for expressing the roughness of the surface. $z_{0}$ is called the roughness length and is usually interpreted as a length scale that characterizes the surface in terms of the efficiency of the process by which momentum is removed from the air stream.

As the surface is approached, additional length scales must enter the 
problem corresponding to the geometrical distribution of the momentum sink, and the wind profile will, in general, depart from a logarithmic shape. Modifying eq. 3 to compensate for the displacement of the momentum sink from the soil surface by introducing the displacement height $d$, such that

$U=\left(\mathrm{u}_{*} / \mathrm{k}\right) \ln \left[(z-d) / \mathrm{z}_{0}\right]$

does not totally eliminate the difficulty, because the vertical distribution of the drag force is still excluded from the problem. Equation 5 must be considered as an empirical modification and, while it may be made to closely match observed wind profiles at relatively small distances above a layer of vegetation, profile analysis based on this equation may not necessarily yield the correct value of surface drag. Nevertheless, $\mathrm{z}_{0}$ and $d$ remain commonly used and descriptive parameters for the roughness and effective level of the underlying surface.

The questions considered here concern the relationships between these surface parameters, the density and vertical distribution of the foliage, and the roughness of the underlying soil surface. A number of workers have sought these relationships based on previously published field data on different types of vegetation or surface roughness. While providing useful information, such reviews are generally limited because details of surface structure are usually missing. For example, Tanner and Pelton (1960) found for agricultural crops that $z_{0}$ could be related to canopy height as $z_{0}=a h^{\mathrm{b}}$, where $a=0.13$ and $b=1.0$. Others have found the constant a to be 0.15 for an artificial crop in a wind tunnel (Plate and Quraishi, 1965) and 0.10 for pliable agricultural crops (Szeicz et al., 1969). This formulation fails to predict any difference in $z_{0}$ between two canopies of the same hiehgt but with different density and spatial distribution of leaves. Lettau (1969) compensated for this by making $z_{0} / h=0.5 s / S$, where $s$ is the silhouette area of the average obstacle and $S$ is the specific area or lot area on the horizontal plane. Lettau suggested that the numerical factor 0.5 corresponds to the average drag coefficient of the individual obstacles. Justification for this relationship was provided by comparisons between predicted and experimentally determined roughness lengths for arrays of bushel-baskets (Kutzbach, 1961) and for fescue grasses.

Lettau was aware that the validity of his proposal is confined to surfaces composed of fairly isolated roughness elements. Surfaces formed by densely packed elements tend to present a smoother aspect to the air above, and to elevate the effective level of drag. One can think of the extreme case of arrays of cubes arranged on a plane. Increasing the density of a sparse array will increase the effective roughness of the surface but, eventually, a skimming flow will be generated in which each cube is 'protected' by upwind obstacles, reducing the total drag force until a new, smooth surface is created at the top of the cubes. There is a range of densities, therefore, in which we expect the effective roughness to decrease with increasing density. For the case of the cubes, this regime would terminate when $s / S=1$ and the cubes fill all the space available, but for vegetation no such limit is imposed. 
For canopies formed by many agricultural crops or forests, it has been observed that $\mathrm{z}_{0} / h$ decreases with increasing leaf area index (LAI) which is the total area of one side of all leaves per unit ground area, and at a rate proportional to $(h-d) / h$ (Thom, 1971). Thus, as the density of the canopy increases and the 'origin' of the above-canopy wind profile becomes a larger fraction of the total height of the vegetation, the effective roughness decreases. The proportionality factor $\lambda$, defined by $z_{0}=\lambda(h-d)$, has been estimated as 0.36 for an artificial crop (Thom, 1971); 0.31 for a wheat crop (Legg and Long, 1975); and 0.3 for a pine forest (Hicks et al., 1975).

Unlike the roughness length, the normalized displacement height $d / h$ should be a monotonic function of canopy density if it is to represent the effective level of drag. Over a wide range of heights, a first approximation to $d$ has been obtained without considering density. For example, Stanhill (1969) fitted the expression $d=a h^{\mathrm{b}}$ to results from 19 different vegetation types, mainly agricultural crops. A linear regression of $\log d$ on $\log h$ resulted in a value of $\mathrm{b}=0.9793$ and $\mathrm{a}=0.702$, when $d$ and $h$ were measured in centimeters. The coefficient of variation was only $6 \%$ and the correlation coefficient was equal to 0.97 . Because b was close to unity, the range of $d / h$ computed by the above expression was small: e.g., $d / h=0.61$ when $h=10 \mathrm{~m}$ and $d / h=0.70$ when $h=0.01 \mathrm{~m}$. Meanwhile, other estimates of the normalized displacement height cover a broader range. For example, Legg and Long (1975) measured $d=0.56 h$ for a wheat crop, while Hicks et al. (1975) measured $d=0.89 h$ for a pine forest.

Assuming Reynolds number similarity (Reynolds number independence), the displacement heights and roughness lengths of canopies of similar geometric properties but of different total heights should scale with $h$ in an identical manner. Only in situations in which boundary layer development is incomplete or when thermal stratification is important, should there be a direct dependence on the absolute height of the vegetation. The height dependence implied by the departure of $b$ from a value of 1 in Stanhill's expression for $d$ results in a dimensional inconsistency, and is surely a consequence of the particular vegetation types selected for the regression fit. Since height is the only variable in Stanhill's expression, differences in $d / h$ resulting from differences in canopy structure and density have been reflected in an apparent height dependence.

\section{COMPUTATIONAL MODELING}

\section{Gradient-diffusion or mixing length models}

Mathematical models of canopy exchange processes in which the vegetation is treated as the vertically-distributed source or sink of heat, water vapor, $\mathrm{CO}_{2}$, or momentum have almost universally relied on gradientdiffusion theory (e.g. Allen, 1975; Inoue, 1977). Solution of the conservation equations requires either the direct input of a height-dependent eddy 
diffusivity, or most often, a method of calculating the diffusivity based on mixing length concepts. The mixing length is specified as a function of the height above the soil surface and the density of the vegetation.

In this section, we show two examples of gradient-diffusion models for the purpose of demonstrating the sensitivity of model results, mean vertical profiles and flux densities, to the prescribed mixing length. Seginer (1974) and Kondo and Akashi (1976) each present results of numerical models of the air flow above and within plant canopies for a range of plant densities. The second authors also show calculations of the effect of changing vertical structure of the canopy.

Both papers indicate that $d / h$ reduces to zero for relatively thin canopies. Seginer shows $d / h=0$ for cah $<0.125$, where $a$ is the leaf area density $\left(\mathrm{m}^{2} \mathrm{~m}^{-3}\right)$ and $a$ is the drag coefficient of the individual canopy elements defined by drag $F=c a U^{2} / 2$. [The factor $\frac{1}{2}$ is included here in accordance with the convention adopted by Seginer.] Kondo and Akashi show $d / h=0$ for cah $<0.6$ in a canopy of vertically uniform density, and cah $<0.5$ for their model B-c canopy which, perhaps, most closely resembles that of an agricultural crop.

The reduction of $d$ to zero for sparse canopies was, in these models, a consequence of the manner in which the mixing length was specified. In both cases, the mixing length $l$ was made to increase linearly with height above the soil surface but was restricted within the canopy to a maximum value imposed by an intrinsic mixing length $l_{\mathrm{i}}$ determined by canopy density. For example, Seginer (1974) assumed that increasing canopy density causes a decrease in $l_{\mathrm{i}}$ and imposed the relationship $\left(l_{\mathrm{i}} / h\right)^{2}(\mathrm{cah})=$ constant. Kondo and Akashi (1976) set their intrinsic mixing length in a manner that was only slightly different, such that $\left(l_{\mathrm{i}} / h\right)(c a h)=$ constant. In sparse canopies, the calculated $l_{i}$ was large enough that no restriction was imposed on the mixing length, which then increased from the soil surface at a rate determined by $l=\mathrm{k} z$. Seginer presented the seemingly plausible argument that in dense canopies, the mixing length increases with decreasing vegetation density due to the increased distance between canopy elements, but when the canopy is progressively thinned, a point is reached beyond which the mixing length is totally controlled by the underlying ground and remains constant. $\mathrm{K}$ theory then predicts a logarithmic wind profile above the canopy but with origin at the soil surface $(d=0)$. The point at which $d$ reduces to zero on progressive thinning coincides with the point at which $l_{\mathrm{i}}$ no longer restricts the mixing length profile. The differences between the two reports with respect to $d / h$, identified in the previous paragraph, result entirely from differences in the methods by which $l_{\mathrm{i}}$ was specified.

The displacement height $d$ is, in fact, predetermined for all canopy densities since it equates with the level at which the above-canopy mixing length extrapolates to zero. Further, the results presented by Seginer show that the displacement height estimated from mixing length concepts is not compatible with the proposition by Thom (1971) that $d$ be identified with the effective 
level of the mean drag on the canopy elements, the center-of-pressure, such that

$d=\left(\frac{\tau_{\mathrm{h}}-\tau_{0}}{\tau_{\mathrm{h}}}\right) \frac{\int_{0}^{h} z F(z) \mathrm{d} z}{\int_{0}^{h} F(\dot{z}) \mathrm{d} z}$

where $\tau_{\mathrm{h}}$ and $\tau_{0}$ are the stresses at the top of the vegetation and at the soil surface, respectively. The term in brackets corrects for the stress at the ground. For example, while the displacement height calculated for the above-canopy logarithmic wind profile reduced to zero at cah $=0.125$ in Seginer's calculations, the level of the resultant drag on the canopy, with shear at the underlying soil surface taken into account, was approximately $0.6 h$.

Because of the arbitrary nature with which $d$ is determined within a gradient-mixing length model, the concept of the coincidence of $d$ with the centre-of-pressure cannot be rejected on the basis of the results by Seginer or by Kondo and Akashi (1976).

The predicted forms of the relationship between $\mathrm{z}_{0} / h$ and $c a h$ are also strongly dependent on the manner in which the mixing length is specified. When the mixing length is made to increase linearly with height above the soil surface but restricted within the height of the vegetation by an intrinsic mixing length $l_{\mathrm{i}}$, and when $l_{\mathrm{i}}$ is fixed, independent of canopy density, $\mathrm{z}_{0} / \mathrm{h}$ increases monotonically with cah (Seginer, 1974). However, when it is assumed that increasing canopy density causes a decrease in $l_{i}$, as previously described, $z_{0} / h$ reaches a maximum value and decreases with further increases in $c a h$ in accordance with the expected unimodal shape.

Seginer's results exhibit a maximum in $z_{0}$ of ca. $0.14 h$ at $c a h \cong 0.15$ for canopies in which plant material is uniformly distributed throughout the canopy height. Kondo and Akashi's results for an equivalent canopy differ considerably. They show $z_{0}$ to reach a peak of about $0.31 \mathrm{~h}$, more than twice that calculated by Seginer, and at a value of $c a h \cong 0.6$, an aerodynamic density 4 times that of the other work.

The studies are consistent though in that $z_{0}$ reaches its maximum value with progressive thinning of the canopy, close to the point at which the calculated displacement height has decreased to zero. Again, this relationship is predetermined by the specification of the mixing length. For all (sparse) canopies in which the mixing length is assumed to be determined only by the height above the soil surface and not restricted by a calculated intrinsic mixing length, $l$ increases according to $l=\mathrm{k} z$ for all $z>0$. The surface is, therefore, the origin of the above-canopy logarithmic wind profile and $d=0$. Within this range, increasing leaf density will result in increased drag for constant mean wind speed at some fixed height $z_{1}>h$, and this will be represented by an increase in $z_{0}$ (see eq. 3). Shortly after the mixing length starts to become limited by the intrinsic mixing length, the effect on $\mathrm{z}_{0}$ is reversed by the increase in $d$. For a fixed $U\left(z_{1}\right), \mathbf{u}_{*}$ must continue to increase as additional plant material is introduced into the canopy (at least 
computationally), but this is overcompensated by the reduction in $z-d$ and $\mathrm{z}_{0}$ decreases thereafter (see eq. 5).

While there are differences in the computed roughness lengths in the Seginer (1974) and Kondo and Akashi (1976) models, these differences are due, primarily, to the manner in which the mixing length is prescribed. Up to $c a h=0.125$, the two models match closely with $z_{0}$ increasing with cah. At this point, the mixing length in the Seginer model becomes limited by the intrinsic canopy value, $d$ increases from zero, $z_{0}$ reaches a peak and declines upon further increases in cah. The mixing length of the Kondo and Akashi model is not limited by the intrinsic mixing length until cah $=0.64$ because of the manner in which $l_{\mathrm{i}}$ is prescribed, and $\mathrm{z}_{0}$ continues to increase to this point before reaching a peak with a magnitude approximately twice that of the other model.

\section{Second-order closure model and procedures}

The examples presented in the previous section demonstrate the shortcomings of gradient-diffusion theory in the relatively complicated environment of the plant canopy. Some of the problems inherent in gradientdiffusion theory are absent or less critical with second-order closure in which some aspects of turbulence are treated explicitly. In second-order closure models, the mean momentum equation is solved in conjunction with conservation equations for the Reynolds stress $\overline{u^{\prime} w^{\prime}}$ and for the velocity variances $\overline{u^{\prime 2}}, \overline{v^{\prime 2}}$, and $\overline{w^{\prime 2}}$. This procedure was applied to the air flow through a uniform stand of vegetation in the belief that computed wind profiles would be less sensitive to model assumptions when the equations are closed at a higher order than when the Reynolds stress is parameterized directly. The numerical canopy flow model was described by Wilson and Shaw (1977), and was used by Pereira and Shaw (1980) to calculate the mean wind profile within the canopy layer for different densities of vegetation and for a range of vertical distribution of leaves. This paper reports the extension of the later study to the above-canopy wind profile.

The equation set and the schemes used to obtain closure are described in Wilson and Shaw (1977), and briefly reviewed in Pereira and Shaw (1980). The closure schemes require the specification of a length scale similar to the canopy mixing lengths adopted by Seginer (1974) and Kondo and Akashi (1976). In the present case, the length scale is used in the parameterization of third moments in the conservation equations such as the triple velocity products, the viscous dissipation, and certain pressure-velocity correlations.

Wind profiles were computed over 80 equally spaced grid intervals from the ground to 4 times the height of the vegetation. At the soil surface, the velocity was set to zero while the stress was calculated from the wind velocity at the first grid point above the surface for a range of surface roughnesses specified by $\mathrm{z}_{0}$ (soil) from $0.001 h$ to $0.0016 h$, which could probably be classified as moderately smooth to moderately rough, respectively. 
Wind profiles were calculated for a range of foliage area indices from 0.25 to 10 . In an aerodynamic sense, the density of the vegetation is specified by the product of the amount of plant material and its drag coefficient, and the plant area density $A$ (previously referred to as the leaf area density $a$ ) is always multiplied by the element drag coefficient $C_{\mathrm{d}}$ in any of the governing equations (we have used the convention $C_{\mathrm{d}}=c / 2$ ).

Since the drag coefficient is difficult to specify because of problems imposed by aerodynamic shading and leaf orientation, it is most general to classify the canopy according to the non-dimensional quantity $C_{\mathrm{d}} A h$. The integral of this over the depth $z / h=0$ to 1 , is equal to the product of the drag coefficient and the plant area index, PAI (total area of one side of all plant material per unit ground area). For convenience, however, an effective drag coefficient of 0.2 was selected as typical for corn (Zea mays L.) (Uchijima and Wright, 1964; den Hartog and Shaw, 1975) and results are expressed both in terms of PAI (assuming $C_{\mathrm{d}}=0.2$ ) and, more generally, in terms of this quantity multiplied by $C_{\mathrm{d}}$, over the range 0.05 to 2 .

The vertical distribution of leaf area in many agricultural crops can be described approximately by a triangular shape. For example, Fig. 1a illustrates that an observed area density profile for corn (Shaw et al., 1974) approaches a simple triangular form quite closely. A wind profile calculated with this triangular distribution was virtually indistinguishable from that calculated using the observed distribution. Other leaf density distributions fit this description to varying degrees; examples of sunflower, wheat, maize, and rice were presented by Monteith (1976).

In consequence, computations presented here are based on a triangular. distribution of canopy elements with the exception that the density at the
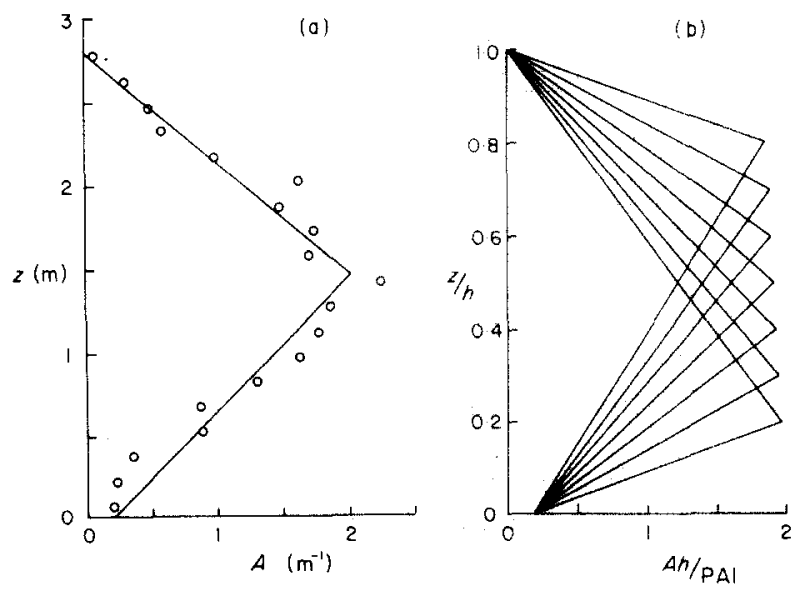

Fig. 1. (a) Plant area density as a function of height for corn (data points), and a triangular distribution with the same total area. (b) Non-dimensional plot of selected plant area densities as functions of height. The area under each curve is equal to 1 . 
surface was one-tenth of the maximum density. The level, $z_{\max }$, at which the maximum density occurred, was made to vary over the range $0.2 h$ to $0.8 \mathrm{~h}$ as shown in Fig. $1 \mathrm{~b}$ in non-dimensional form, in an attempt to mimic leaf density profiles of corn at other stages of morphology and of other crop stands. No attempt was made to represent stands of more vertically homogeneous density, or of forest-like stands with a relatively open trunk space.

Computed mean wind velocity profiles from above the canopy were analyzed in the usual manner for the displacement height and the roughness length, using eq. 5 but replacing wind speed $U$ by velocity $\overline{\mathbf{u}} . \mathbf{u}_{*} / \mathrm{k}$ was treated as an unknown quantity. Profiles of $z_{0}$ and $d$ could be calculated locally from three consecutive grid points. Both were found to vary with height reflecting the fact that the computed wind profile was not logarithmic immediately above the vegetation. For this reason, $d$ was also calculated as the center-of-pressure according to eq. 6 , and $\mathrm{z}_{0}$ was calculated using this value of $d$ and the normalized wind velocity $\overline{\mathbf{u}} / \mathbf{u}_{*}$ computed at $z / h=2$.

\section{RESULTS AND DISCUSSION}

Comparisons of displacement heights calculated from: (a) the computed wind profile at twice the canopy height; (b) a gradient-diffusion or length model; and (c) the center-of-pressure from the computed in-canopy wind profile, are shown in Fig. 2 over a range of plant area indices for canopies of vertically uniform density and for canopies with a triangular distribution of leaves with maximum density at $0.5 h$.

In each case, the displacement height calculated by analysis of the computed wind profile was a fairly close match for the center-of-pressure within

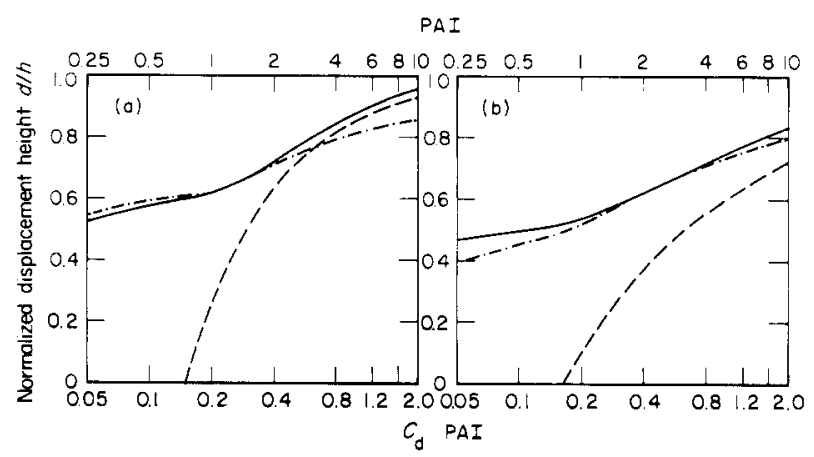

Fig. 2. Displacement height calculated from the computed wind profile at $2 h$ (dash/dot curve); a gradient-diffusion/mixing length model (dashed curve); and from the centerof-pressure (solid curve) as a function of $C_{\mathrm{d}} \cdot P A I$ (and against $P A I$ assuming that $C_{\mathrm{d}}=$ 0.2 ) for (a) canopies of vertically uniform density, and (b) a triangular distribution of leaf area with maximum density at $h / 2$. All curves are hand-drawn through the computed points. 
the canopy, while gradient-diffusion theory results in a value of $d$ that progressively diverges from the other two values, with decreasing canopy density, until reaching zero at $C_{\mathrm{d}} \cdot P A I \cong 0.16$ in each case. This aerodynamic density is equivalent to $c a h \cong 0.32$, a value intermediate between those of the Seginer (1974) and Kondo and Akashi (1976) models discussed earlier.

The mixing length of the gradient-diffusion model and the length scale of the second-order model were defined in an identical fashion as a linear function of height above the ground but restricted so as not to exceed an intrinsic value within the canopy. Yet, the results are quite different. The gradient-diffusion model automatically creates a logarithmic wind profile above the canopy with a displacement height equal to the height at which the above-canopy mixing length extrapolates to zero. On the other hand, the second-order model allows the profile to deviate from the logarithmic shape near the canopy, but calculations of $d$ made at twice the canopy height are close to the center-of-pressure over a wide range of aerodynamic densities.

Thom (1971) asserts that the displacement height can be identified with the center-of-pressure of the drag force within the canopy but this has not been verified experimentally over a wide range of plant densities. A major problem is the difficulty of determining $d$ from measured mean wind profiles since $d$ depends primarily on the second derivative of wind speed with respect to height (the curvature of the profile) and is thus very sensitive to experimental error.

There is logic to Thom's proposition, however, and the current results lend additional support. Meanwhile, it is difficult to defend the gradient-

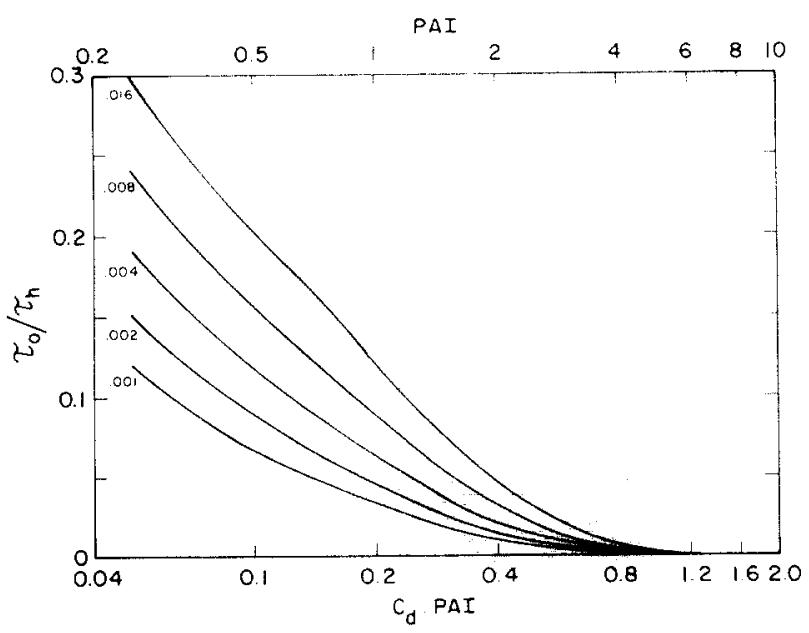

Fig. 3. Ratio of the stress at the soil surface $\tau_{0}$ to the total stress $\tau_{h}$ for a range of soil roughness lengths indicated as $z_{0}$ (soil/h on the diagram, and as a function of $C_{\mathrm{d}} \cdot P A I$ (and against $P A I$ assuming that $C_{\mathrm{d}}=0.2$ ). All curves are hand-drawn through the computed points. 
diffusion model conclusion that the origin of the logarithmic wind profile descends to the surface for relatively sparse canopies, when the center of the drag force is at mid-canopy and the soil surface still plays a minor role in the overall drag. The center of the drag force is shown as the center-of-pressure in Fig. 2 and the relative contribution of the surface to the above-canopy shear stress is illustrated in Fig. 3 based on calculations from the secondorder closure model. The latter diagram shows that for rough soil surfaces and very thin canopies, the soil accounted for as much as $30 \%$ of the total drag, but that normally the stress at the soil is a minor contribution.

In its present form, the second-order closure model is not capable of predicting the height to which a significant departure from the logarithmic profile should be observed. The model does illustrate that such a departure will occur. The problem appears to lie with the parameterization of the third-order transport terms in the second-moment equations, to which the height of the canopy influence and the calculated profile of displacement height appear to be particularly sensitive. For this reason, the displacement height shown in the remaining diagrams is the center-of-pressure calculated from computed wind profiles according to eq. 6 .

Figure 4 shows that calculated displacement heights increased monotonically with increasing density of the vegetation, and as the level of maximum leaf density $\left(z_{\max } / h\right)$ moved upward in the canopy. Both results are to be expected; as the density of the vegetation increases and as the bulk of the material is oriented towards the top of the canopy, the vertical profile of the drag force should be displaced towards the upper part of the canopy.

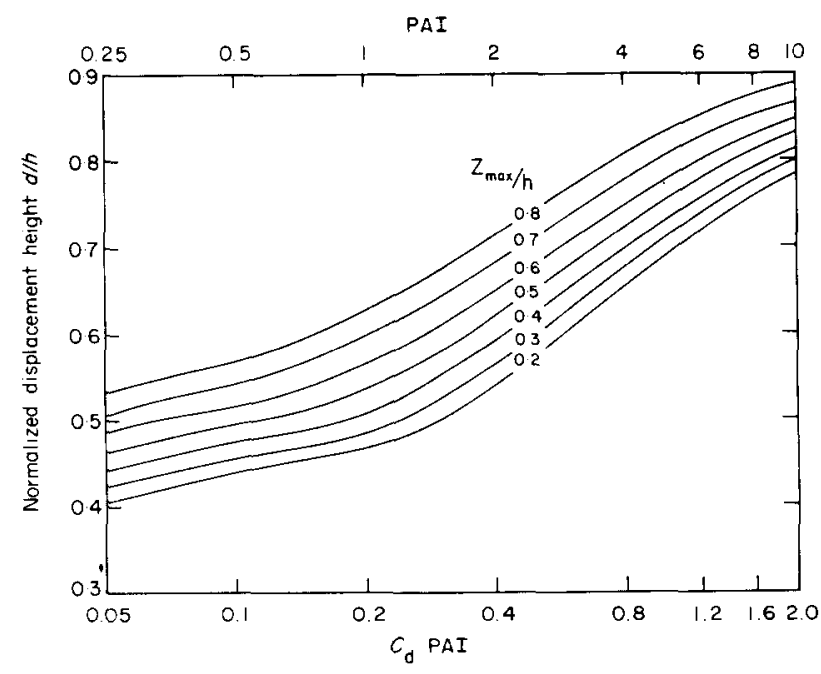

Fig. 4. Normalized displacement height as a function of $C_{\mathrm{d}} \cdot P A I$ (and against $P A I$ assuming that $C_{\mathrm{d}}=0.2$ ). All curves are hand-drawn through the computed points and are labelled according to the height at which density reaches a maximum. 


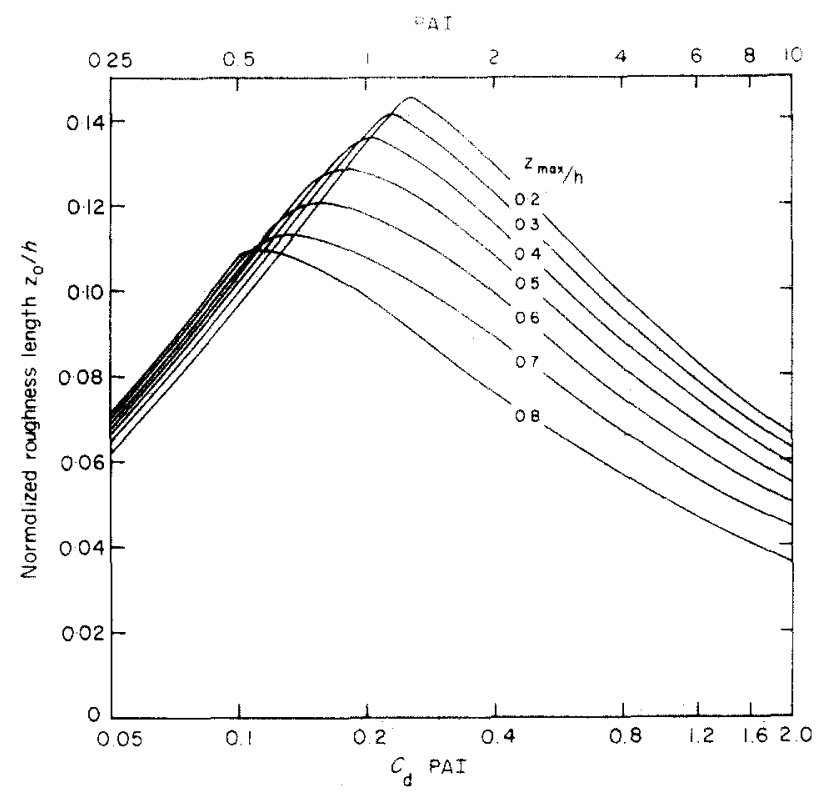

Fig. 5. Normalized roughness length as a function of $C_{\mathrm{d}} \cdot P A I$ (and against $P A I$ assuming that $C_{\mathrm{d}}=0.2$ ). All curves are hand-drawn through the computed points and are labelled according to the height at which density reaches a maximum.

The relationship between roughness length and canopy density is shown in Fig. 5 . Each curve is unimodal. At low area indices, $z_{0}$ increased with increasing density as predicted by the Lettau (1969) relationship, and the roughest surfaces were those in which plant material was skewed towards the top of the canopy. This result indicates that when the vegetation is relatively sparse, the greatest drag is achieved when plant material is projected to the upper part of the canopy where wind speeds are greatest.

The roughness length reached a peak value at an aerodynamic density $(C d \cdot P A I)$ which depended on the vertical structure of the canopy but which was in the range 0.1 to 0.24 ( $c a h$ in the range 0.2 to 0.48 ). Further increases in density produced a decrease in the calculated roughness length. For these denser canopies, $z_{0}$ was very dependent on canopy structure and largest when leaf area was skewed toward the bottom of the canopy. In this range of canopy density, the surface of the vegetation is least rough when most of the plant material is near the top of the canopy, tending to seal the canopy aerodynamically and to present a smoother surface to the air flow above. On the other hand, when the canopy is relatively open at the top, momentum penetrates more easily and a greater leaf area is presented to the force of the wind producing an effectively greater roughness.

Figure 6 shows the relationship between the displacement heights and roughness lengths calculated from computed wind profiles. The aerodynamic density and the canopy structure $\left(z_{\max } / h\right)$ are identified where possible but 


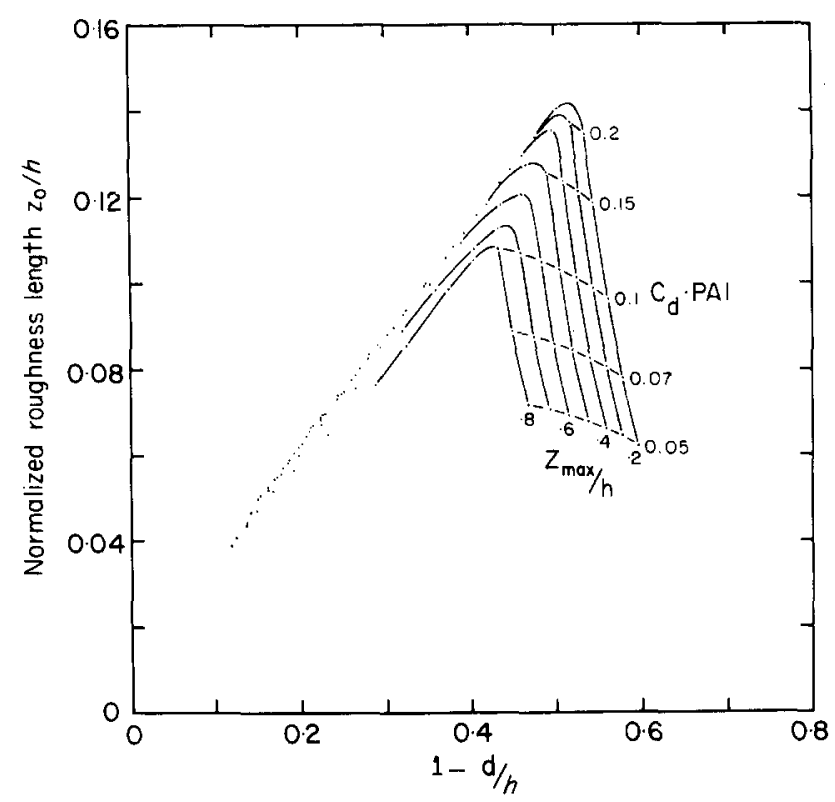

Fig. 6. Plot of roughness length against displacement height. $C_{\mathrm{d}} \cdot P A I$ and height of maximum density are identified where possible.

generally, when $C d \cdot P A I$ exceeded about $0.2($ cah $\simeq 0.4)$ the calculated points fell close to a straight line. This line does not appear to pass through the origin but has a $y$-intercept of about 0.01 and a slope of about 0.26 . A line forced through the origin to satisfy the equation $z_{0} / h=\lambda(1-d / h)$ and passing through intermediate values of $d / h$ of about 0.7 has a slope $\lambda \simeq 0.29$. This is close to the experimental value quoted earlier for real and artificial crops and for a forest.

\section{SUMMARY AND CONCLUSIONS}

A second-order closure model has been used to compute wind profiles within and above plant canopies of different density and structure to observe effects on the roughness length and the displacement height. The belief that the computed wind field will be less sensitive to the parameterization of the third moments in the governing equations, than to the parameterization of the momentum flux directly, is supported by the present results. This support is illustrated by the comparison made between results of models based on gradient-diffusion theory (Seginer, 1974; Kondo and Akashi, 1976) and the second-order model described by Wilson and Shaw (1977). It was shown that the form of the relationships between $\mathrm{z}_{0}, d$, and the aerodynamic density of the vegetation is strongly dependent on the manner in which the mixing length is prescribed in a gradient-diffusion model. In fact, the displacement height is forced to zero when canopy density decreases to a point 
where the mixing length is thought to be determined only by the distance from the soil surface. This occurs when the effective level of the drag forces (the center-of-pressure) is still a relatively large fraction of the canopy height and when the soil surface is playing a very minor role in the overall drag.

The closure schemes of the second-order model also utilized a length scale that was restricted within the canopy according to canopy density. Parameterizations using the prescribed length scale were applied only to thirdorder terms in the governing equations and did not force the displacement height to zero for sparse canopies when the length scale was assumed to increase linearly from the soil surface. In fact, calculated values of the displacement height approximated the center-of-pressure over the complete range of densities examined.

Based on the assumption that, ideally, calculated results should be independent of input variables that can be assigned arbitrarily, we interpret the results discussed above to indicate that the second-order model represents the canopy flow situation to a better approximation than does a gradientdiffusion model.

Displacement height and roughness length were examined by analyzing computed wind profiles for a range of aerodynamic densities and for different vertical distributions of vegetation density throughout the depth of the canopy. Increasing density resulted in a monotonic increase in displacement height while roughness length exhibited a unimodal relationship with plant density. Roughness length initially increased with increasing density, reached a peak, and then declined. The form of this relationship was dependent on canopy structure since, for sparse canopies, greatest roughness occurred when the center of gravity of the canopy was near the top of the canopy while, in dense canopies, greatest roughness occurred when the center of gravity was near the bottom of the canopy. Growing crop canopies will, in general, pass through this maximum in $z_{0} / h$ during their growth cycle, but whether or not the absolute value of $z_{0}$ peaks and then declines will depend on the particular growth pattern of the crop and the relationship between increase in height and increase in leaf area density with time.

Within the range in which $z_{0}$ decreased with increasing density, there was a unique relationship between $z_{0}$ and $d$. When $z_{0} / h$ was plotted against $1-d / h$, calculated points fell close to a straight line, regardless of the structure or density of the canopy.

This straight line did not pass through the origin as expected, but crossed the $z_{0} / h$ axis at a value of about 0.01 . A line forced through the origin to pass through points having a displacement height approximately $70 \%$ of the canopy height had a slope of about 0.29 which agrees well with experimental values quoted elsewhere.

\section{ACKNOWLEDGMENTS}

This study was conducted partly with the support of the National Science Foundation under Grant No. NSF-ATM-8017915. One of the authors (A.R.P.) received financial support from Empresa Brasileira de Pesquisa Agropecuaria. 


\section{REFERENCES}

Allen, L.H., Jr., 1975. Line source carbon dioxide release: Predictions by a two-dimensional numerical diffusion model. Boundary-Layer Meteorol., 8: 39-79.

Den Hartog, G. and Shaw, R.H., 1975. A field study of atmospheric exchange processes within a vegetative canopy. In: D.A. DeVries and N.H. Afgan (Editors), Heat and Mass Transfer in the Biosphere. Scripta Books, Washington, DC, pp. 299-309.

Hicks, B.B., Hyson, P. and Moore, C.J., 1975. A study of eddy fluxes over a forest. J. Appl. Meteorol., 14:58-66.

Inoue, K., 1977. Numerical experiments about three-dimensional transfer of $\mathrm{CO}_{2}$ over a finite model rice field in relation to canopy photosynthesis. Bull. Natl. Inst. Agric. Sci., Ser. A, $24: 19-44$.

Kondo, J. and Akashi, S., 1976. Numerical studies on the two-dimensional flow in horizontally homogeneous canopy layer. Boundary-Layer Meteorol., 10: 255-272.

Kutzbach, J.E., 1961. Investigations of the modification of wind profiles by artificially controlled surface roughness. Ann. Rep. Dep. Meteorol., Univ. of Wisconsin, Madison, WI, Ann. Rep. No. DA-36-039-SC-80282, pp. 71-113.

Legg, B.J. and Long, I.F., 1975. Turbulent diffusion within a wheat canopy: II. Results and interpretation. Q.J.R. Meteorol. Soc., 101:611-628.

Lettau, H., 1969. Note on aerodynamic roughness-parameter estimation on the basis of roughness-element description. J. Appl. Meteorol, , 8: 828--832.

Monteith, J.L., 1976. Vegetation and the Atmosphere. Vol. 2. Academic Press, New York, NY, $439 \mathrm{pp}$.

Pereira, A.R. and Shaw, R.H., 1980. A numerical experiment on the mean wind structure inside canopies of vegetation. Agric. Meteorol., 22: 303-318.

Plate, E.J. and Quraishi, A.A., 1965. Modeling of velocity distributions inside and above tall crops. J. Appl. Meteorol., 4: 400-406.

Seginer, I., 1974. Aerodynamic roughness of vegetated surfaces. Boundary-Layer Meteorol., $5: 383-393$.

Shaw, R.H., den Hartog, G., King, K.M. and Thurtell, G.W., 1974. Measurements of mean wind flow and three-dimensional turbulence intensity within a mature corn canopy. Agric. Meteorol., 13: 419--425.

Stanhill, G., 1969. A simple instrument for the field measurement of turbulent diffusion flux. J. Appl. Meteorol., 8: 509-513.

Szeicz, G., Endrödi, G. and Tajchman, S., 1969. Aerodynamic and surface factors in evaporation. Water Resour. Res., 5: 380-394.

Tanner, C.B. and Pelton, W.L., 1960. Potential evapotranspiration estimates by the approximate energy balance method of Penman. J. Geophys. Res., $65: 3391-3413$.

Thom, A.S., 1971. Momentum absorption by vegetation. Q.J.R. Meteorol. Soc., 97: $414-428$.

Uchijima, Z. and Wright, J.L., 1964. An experimental study of airflow in a corn plant-air layer. Bull. Natl. Inst. Agric. Sci., Ser. A, 11:19-66.

Wilson, N.R. and Shaw, R.H., 1977. A higher-order closure model for canopy flow. J. Appl. Meteorol., 16: 1197-1205. 